\title{
ANÁLISE DOS FATORES DE RISCO PARA GAGUEIRA EM CRIANÇAS DISFLUENTES SEM RECORRÊNCIA FAMILIAL
}

\author{
Analysis of the risk factors for stuttering in disfluent children \\ without familial recurrence
}

\author{
Cristiane Moço Canhetti de Oliveira ${ }^{(1)}$, Heloisa Aparecida de Souza ${ }^{(2)}$, \\ Ana Claudia dos Santos ${ }^{(3)}$, Denise de Souza Cunha ${ }^{(4)}$
}

\begin{abstract}
RESUMO
Objetivo: analisar os fatores gênero, idade, tipo de surgimento da gagueira, tempo de duração e tipologia das disfluências, fatores estressantes físicos e emocionais, e fatores comunicativos e qualitativos associados em crianças disfluentes sem recorrência familial do distúrbio. Método: participaram 43 crianças com alto risco para a gagueira de ambos os gêneros. A coleta de dados foi realizada por meio do Protocolo de Risco para a Gagueira do Desenvolvimento - PRGD. Resultados: a razão masculino/feminino foi de 3,3:1. A única diferença estatisticamente significante dos fatores de risco analisados nos gêneros masculino e feminino foi a maior ocorrência de fatores comunicativos associados no gênero masculino $(p=0,003)$. Houve uma semelhança dos achados entre os meninos e as meninas: quanto ao tempo de duração das disfluências a maioria apresentou mais de 12 meses de duração, a tipologia gaga foi a mais freqüente, a presença de fatores estressantes emocionais ocorreu na maior parte das crianças, e finalmente os fatores qualitativos associados, como taxa de elocução aumentada, tensão visível e incoordenação pneumo-fono-articulatória estiveram presentes em grande parte da amostra. Conclusão: os resultados desta investigação permitiram concluir que nos casos de crianças com alto risco para a gagueira isolada ocorreu a interação de inúmeros fatores, sugerindo que o distúrbio é multifatorial. Também foi possível concluir que a interação de alguns fatores como gênero masculino, tipologia gaga manifestada por mais de 12 meses, com início persistente, na presença de fatores qualitativos e comunicativos associados pode representar risco maior para o desenvolvimento da gagueira persistente.
\end{abstract}

DESCRITORES: Fonoaudiologia; Fala; Gagueira; Fatores de Risco; Distúrbios da Fala

\section{INTRODUÇÃO}

A gagueira é um distúrbio da fala que resulta de uma influência complexa de múltiplos fatores

(1) Fonoaudióloga; Docente do Departamento de Fonoaudiologia da Faculdade de Filosofia e Ciências da Universidade Estadual Paulista, UNESP, Marília, SP, Brasil; Doutora em Ciências Biológicas na área de Genética pelo Instituto de Biociências da Universidade Estadual Paulista, UNESP, Botucatu, SP.

(2) Fonoaudióloga graduada pela Faculdade de Filosofia e Ciências da Universidade Estadual Paulista, UNESP, Marília, SP, Brasil.

(3) Graduanda em Fonoaudiologia pela Faculdade de Filosofia e Ciências da Universidade Estadual Paulista, UNESP, Marília, SP, Brasil.

(4) Graduanda em Fonoaudiologia pela Faculdade de Filosofia e Ciências da Universidade Estadual Paulista, UNESP, Marília, SP, Brasil.

Conflito de interesses: inexistente que incluem a predisposição genética, habilidades motoras da fala, fatores lingüísticos, cognitivos, emocionais e ambientais ${ }^{1}$. Para os estudiosos ${ }^{1}$, o peso de cada fator e como eles interagem com outros fatores no tempo, provavelmente resultam em diferenças consideráveis entre indivíduos com gagueira. Sabe-se também que o espectro dos fatores de risco na gagueira é amplo e heterogêneo².

A implicação clínica deste conhecimento é que para promover uma melhor intervenção às crianças que gaguejam, é necessário delinear e direcionar os fatores de risco mais relevantes, pois a definição do quadro apresentado pela criança irá nortear a melhor conduta terapêutica a ser utilizada, e conseqüentemente favorecer a promoção da fluência. Crianças pequenas respondem melhor a terapia prevenindo a evolução do distúrbio para 
um quadro mais crônico e com pior prognóstico ${ }^{3,4}$. Portanto, o diagnóstico e a intervenção precoce são importantes, e por isso os fatores de risco para a gagueira desenvolvimental persistente devem ser investigados ${ }^{5,6}$.

Quanto aos fatores genéticos da gagueira, por exemplo, a literatura aponta para a existência de alguns subtipos de gagueira ${ }^{7,8}:$ um de origem genética, onde ocorre a recorrência familial do distúrbio, e; outro composto por casos esporádicos ou isolados, não ocorrendo a recorrência familial, podendo ser decorrentes de danos cerebrais precoces ${ }^{9,10}$.

Em alguns casos pode ocorrer a dificuldade em diferenciar crianças que irão se recuperar espontaneamente daquelas que irão desenvolver gagueira crônica, por isso, as informações sobre os fatores de risco para a cronicidade da gagueira são necessárias. Dentre estes fatores, estão o gênero, a idade, o tipo de surgimento da gagueira, o tempo de duração e a tipologia das disfluências, os fatores estressantes físicos ou o histórico mórbido pré, peri e pós natal, o histórico familial, os fatores estressantes emocionais que ocorreram próximo ao surgimento do distúrbio, bem como os fatores comunicativos e qualitativos associados ${ }^{11}$.

O gênero da criança, entre outros fatores, influencia na prevalência da gagueira, pois o maior risco para o desenvolvimento deste distúrbio ocorre em indivíduos do gênero masculino, sendo que esta proporção aumenta com a idade ${ }^{10,12-15}$. A distribuição sexual da gagueira infantil acontece na mesma proporção em ambos os sexos para crianças de 2 a 3 anos $^{12}$, e à razão de 2,1 meninos: 1 menina para crianças de 2 a 6 anos $^{16}$, o que demonstra que a prevalência sexual da gagueira no gênero masculino está presente desde o período inicial do distúrbio.

Considerando a idade, as disfluências frequentemente aparecem nos anos pré-escolares ${ }^{17-20}$, com uma idade média do surgimento de 30 meses $^{21}$. Dessa forma, é durante os anos pré-escolares que ocorre o início das disfluências gagas, prejudicando a comunicação verbal e, muitas vezes, sendo acompanhado de sentimentos e emoções negativas como timidez, medo relacionado à fala $e$ ansiedade em determinadas situações ${ }^{22}$.

O surgimento persistente da gagueira (quando o distúrbio surgiu desde o início da fala, permanece constante e vai se agravando com o tempo) e um tempo maior de 12 meses de duração das disfluências é indicativo de um distúrbio crônico ${ }^{11}$. A recuperação espontânea da gagueira ocorre principalmente nos primeiros anos de vida, sendo que muitas crianças se recuperaram dentro dos 12 meses após o início das disfluências ${ }^{16,23}$. Portanto, quanto mais nova a criança e quanto menor o tempo de duração das disfluências, maior a possibilidade de ocorrer a recuperação espontânea da gagueira.

O conhecimento dos diferentes tipos de disfluências é fundamental para o diagnóstico, pois a presença em quantidade aumentada de disfluências gagas é indicativo de gagueira. Sabe-se que freqüentemente as disfluências que ocorrem dentro da palavra são consideradas disfluências gagas, como bloqueio, prolongamentos, repetições de sons ou de parte de palavras. As disfluências mais comumente encontradas na gagueira são repetições de sons ou sílabas e posição articulatória fixa, resultando em bloqueios ou prolongamentos de sons $^{24,25}$. As disfluências comuns freqüentemente ocorrem entre as palavras, como hesitações, interjeições e revisões. As repetições podem ser tanto comuns ou gagas dependendo da unidade que foi repetida; quando a unidade for pequena, como uma palavra monossilábica, parte da palavra ou som, é considerada como disfluência gaga. Repetições de unidades grandes como palavras inteiras não monossilábicas, parte do enunciado ou de frases são consideradas como disfluências comuns.

A integridade do sistema nervoso, bem como os aspectos orgânicos são pré-requisitos para uma fala fluente. Segundo alguns estudiosos ${ }^{26}$, o sintoma mais óbvio e primário da gagueira é a falha intermitente do sistema nervoso de gerar sinais que comandam apropriadamente os músculos daquelas atividades que devem ser controladas dinamicamente para a fala fluente ser produzida. Neste sentido, os fatores estressantes físicos que podem ocorrer no período do desenvolvimento infantil, como danos cerebrais precoces, traumas ou doenças no nascimento podem colaborar para a manifestação da gagueira.

$\mathrm{Da}$ mesma forma, os fatores psicossociais estressantes como problemas nas relações familiares ou sociais, mortes de parentes próximos, separação dos pais que ocorreram próximo ao surgimento das disfluências podem colaborar para o início da gagueira. Estes fatores podem causar e aumentar a ansiedade das crianças, e segundo alguns estudiosos, a ansiedade é um dos muitos fatores que podem predispor, precipitar ou manter a gagueira ${ }^{27}$.

Apesar de a literatura descrever quais são os fatores de risco para a gagueira persistente, investigações que analisaram a relação entre esses fatores, bem como quais associações de fatores são consideradas mais preocupantes para o desenvolvimento da gagueira crônica são escassas. Outra lacuna que existe na literatura nesta área é a descrição dos fatores de risco em determinados subtipos de gagueira, como a gagueira isolada. 
Deste modo, o objetivo deste trabalho foi analisar os fatores gênero, idade, tipo de surgimento da gagueira, tempo de duração e tipologia das disfluências, os fatores estressantes físicos e emocionais, e os fatores comunicativos e qualitativos associados em crianças disfluentes sem recorrência familial do distúrbio.

\section{MÉTODO}

O presente estudo foi realizado com 43 crianças com alto risco para a gagueira, cujos pais e/ou professores apresentaram queixa de gagueira e procuraram o atendimento fonoaudiológico no Programa de Intervenção na Disfluência Infantil - PIDI ou no Estágio Supervisionado de Terapia Fonoaudiológica: Fluência, da UNESP/ Marília. As crianças foram inclusas sem distinção de gênero, escolaridade ou nível sócio-econômicocultural. O grupo foi formado por 43 crianças com alto risco para a gagueira na faixa etária de 3.0 a 11.11 anos de idade (média 5,79 anos e DP=21,98) de ambos os gêneros (33 do gênero masculino e 10 do gênero feminino).

Os requisitos de inclusão dos participantes foram: idade entre 3 anos e 11 anos e 11 meses, ser falante nativo do português brasileiro, apresentar queixa de gagueira, não apresentar histórico familial positivo para a gagueira (mesmo em familiares de segundo e terceiro graus), e maior pontuação na coluna alto riso para a cronicidade da gagueira no Protocolo de Risco para a Gagueira do Desenvolvimento - PRGD ${ }^{11}$.

Os critérios de exclusão dos participantes foram: apresentar qualquer distúrbio neurológico, genético ou não, tais como distonia, doenças extras piramidais, deficiência mental, epilepsia, transtorno do déficit de atenção e hiperatividade (TDAH); sintomas ou condições psiquiátricas; apresentar alterações de comunicação oral não compatíveis com a idade, com exceção de gagueira; apresentar perda auditiva condutiva ou neurossensorial, e outras condições pertinentes que poderiam gerar erros no diagnóstico.

Inicialmente os familiares dos participantes receberam informações sobre os objetivos do estudo e explicação dos procedimentos que foram realizados, e deram consentimento, por escrito, para a participação na pesquisa. A coleta de dados foi realizada por meio preenchimento do Protocolo de Risco para a Gagueira do Desenvolvimento PRGD ${ }^{11}$. O Protocolo foi realizado com os pais ou familiares das crianças com a finalidade de detectar os fatores de risco para a cronicidade da gagueira. Dados específicos sobre as disfluências foram pesquisados, como gênero, idade, tipo de surgimento da gagueira, tempo de cronicidade e tipologia das disfluências, fatores estressantes físicos e/ou emocionais que ocorreram próximo ao surgimento das disfluências, e fatores comunicativos e qualitativos associados.

Os dados dos antecedentes familiais para realizar o heredograma foram coletados no item sobre o histórico familial. Os familiares adultos de primeiro grau (pai ou mãe) foram questionados sobre o padrão de fluência de seus parentes e sobre a existência de alguém na família que eles soubessem apresentar gagueira. Para possibilitálos a responderem estas questões, a entrevistadora apresentou uma definição padronizada de gagueira dando exemplos que pudessem ilustrá-la. Gagueira foi definida como "interrupção na continuidade do fluxo da fala caracterizada como repetições, prolongamentos, ou bloqueios de sons, sílabas ou de pequenas palavras"12. Exemplos de repetições de sons ou de sílabas, repetições de palavras monossilábicas, prolongamentos de sons, bloqueios e intrusões foram oferecidos. Com o intuito de obter informações precisas, os familiares foram encorajados a checar e a confirmar com outros parentes, informações e histórias de gagueira.

O estudo foi iniciado após aprovação do Comitê de Ética em Pesquisa da Faculdade de Filosofia e Ciências da UNESP - Parecer CEP № 1784/2009, e a coleta dos dados após a autorização dos participantes, obtida por meio do Termo de Consentimento Livre e Esclarecido.

Os dados foram submetidos à análise estatística por meio dos Testes Mann-Whitneye Qui-quadrado, ajustado pela Estatística de Fisher. O nível de significância adotado foi de 5\% para todos os testes.

\section{RESULTADOS}

A média de idade da população estudada foi de $5,79 \pm 1,98$ anos, estando $69,8 \%$ na faixa etária de 5 a 10 anos. Inicialmente a análise dos dados foi realizada por meio de uma comparação dos fatores de risco apresentados pelo gênero masculino e feminino. A razão masculino/feminino foi de 3,3:1. A análise estatística aponta que não houve diferença estatisticamente significante entre os grupos para a idade (Tabela 1). 
Tabela 1 - Comparação da idade das crianças entre o gênero masculino e feminino

\begin{tabular}{cccccccc}
\hline Variável & Grupos & N & Média & $\begin{array}{c}\text { Desvio- } \\
\text { padrão }\end{array}$ & Mínimo & Máximo & $\begin{array}{c}\text { Significância } \\
(\mathbf{p})\end{array}$ \\
\hline \multirow{3}{*}{ Idade } & Masculino & 33 & 5,61 & 1,66 & 3,00 & 9,00 & 0,590 \\
\cline { 2 - 8 } & Feminino & 10 & 6,40 & 2,84 & 3,00 & 11,00 & 0,590 \\
\cline { 2 - 8 } & Total & 43 & 5,79 & 1,98 & 3,00 & 11,00 & \\
\hline
\end{tabular}

Teste de Teste de Mann-Whitney

*Valores estatisticamente significantes $(p \leq 0,005)$

A Tabela 2 apresenta os resultados referentes aos vários fatores de risco analisados separadamente por gênero e também por grupo, com o número e porcentagem para cada tipo de resposta obtida. A única diferença estatisticamente significante foi a maior ocorrência de fatores comunicativos associados no gênero masculino, em relação ao feminino $(p=0,003)$. Os meninos apresentaram significantemente maior quantidade de fatores comunicativos associados.

Porém, vale ressaltar algumas tendências de divergências em relação a alguns fatores analisados nos dois gêneros. Nota-se que quanto à idade houve mais meninas na faixa etária de 3 a 4 anos em relação aos meninos. Quanto ao tipo de surgimento as meninas apresentaram mais freqüentemente o tipo súbito em relação aos meninos. Os fatores estressantes físicos ocorreram mais freqüentemente no gênero masculino em relação ao feminino.

Nos outros fatores analisados, houve uma semeIhança dos achados entre os meninos e as meninas: quanto ao tempo de duração das disfluências a maioria apresentou mais de 12 meses de duração, a tipologia gaga foi a mais freqüente, a presença de fatores estressantes emocionais ocorreu na maior parte das crianças, e finalmente os fatores qualitativos associados, como taxa de elocução aumentada, tensão visível e incoordenação pneumo-fonoarticulatória estiveram presentes em grande parte da amostra.

$\mathrm{Na}$ análise das crianças com tipologia gaga ( $\mathrm{N}=26,60,40 \%$ da amostra total) verifica-se que $92,30 \%$ apresentaram as disfluências por mais de 12 meses, 76,92\% tinham algum fator qualitativo associado e $61,53 \%$ manifestaram pelo menos um fator comunicativo associado (Tabela 3 ).

$\mathrm{Na}$ Tabela 4 pode-se observar que a maioria das crianças $(n=24,55,80 \%)$ apresentou ausência de fatores estressantes físicos e presença de fatores estressantes emocionais. A análise estatística realizada não mostrou diferença estatisticamente significante entre esses fatores.

\section{DISCUSSÃO}

A literatura ${ }^{19,26}$ sobre a gagueira desenvolvimental familial é vasta, tendo em vista a procura dos genes candidatos à transmissão do distúrbio. Porém, poucas investigações se dedicam a analisar o subgrupo de gagueira isolada. Sendo assim, esta pesquisa teve como objetivo analisar os fatores de risco para a gagueira num subgrupo de crianças com alto risco para a gagueira isolada.

A razão sexual encontrada nesta pesquisa (3.3M:1F) mostra a maior prevalência da gagueira no gênero masculino, evidenciando que estas crianças apresentam um risco maior para o distúrbio quando comparado com o gênero feminino, conforme descrito na literatura ${ }^{10,12-15}$.

Quando a amostra foi separada por gênero masculino e feminino, não houve diferença estatisticamente significante com relação à idade, tipo de início e tipologia das disfluências, tempo de cronicidade das disfluências, fatores estressantes físicos e emocionais e fatores qualitativos associados. No entanto, os dados indicaram que esses fatores, juntos, podem contribuir para o surgimento e persistência da gagueira, independente do gênero enfatizando a natureza multidimensional do distúrbio e confirmando estudos prévios ${ }^{1,2,8,23}$.

A única diferença significante entre os gêneros foi com relação aos fatores comunicativos associados, que ocorreram em maior quantidade nos disfluentes do gênero masculino. Esta evidência corrobora os achados da literatura que relatam que crianças com algum distúrbio desenvolvimental relacionado à fala apresentam um risco maior para desenvolver a gagueira ${ }^{28,29}$, e que crianças com gagueira persistente apresentaram pior desempenho na avaliação fonológica e de linguagem do que crianças com gagueira recuperada ${ }^{23}$.

No entanto, nossos achados sugerem que esse risco é aumentado de forma significante quando esta criança é do gênero masculino, independente da predisposição genética para o distúrbio. Porém, a presença do histórico familial positivo para o 
Tabela 2 - Distribuição dos participantes segundo os fatores de risco analisados, como um grupo e separados de acordo com o gênero

\begin{tabular}{|c|c|c|c|c|c|c|c|}
\hline \multirow[t]{2}{*}{ Fatores de risco } & \multicolumn{2}{|c|}{$\begin{array}{l}\text { Gênero masculino } \\
(\mathrm{N}=33)\end{array}$} & \multicolumn{2}{|c|}{$\begin{array}{l}\text { Gênero feminino } \\
\qquad(N=10)\end{array}$} & \multicolumn{2}{|c|}{$\begin{array}{c}\text { Total } \\
(\mathrm{N}=43)\end{array}$} & \multirow[t]{2}{*}{ Valor de $p$} \\
\hline & $\mathbf{N}$ & $\%$ & $\mathbf{N}$ & $\%$ & $\mathbf{N}$ & $\%$ & \\
\hline \multicolumn{8}{|l|}{ Idade } \\
\hline$\geq 3$ e $<4$ & 5 & 15,20 & 4 & 40,00 & 9 & 20,90 & \multirow{4}{*}{0,169} \\
\hline$\geq 5$ e $<7$ & 14 & 42,40 & 1 & 10,00 & 15 & 34,90 & \\
\hline$\geq 7$ e $<10$ & 11 & 33,30 & 4 & 40,00 & 15 & 34,90 & \\
\hline$\geq 10$ e $<12$ & 3 & 9,10 & 1 & 10,00 & 4 & 9,30 & \\
\hline \multicolumn{8}{|l|}{ Tipo de surgimento } \\
\hline Súbito & 4 & 12,10 & 4 & 40,00 & 8 & 18,60 & \multirow{3}{*}{0,132} \\
\hline Cíclico & 11 & 33,33 & 2 & 20,00 & 13 & 30,23 & \\
\hline Persistente & 18 & 54,50 & 4 & 40,00 & 22 & 51,17 & \\
\hline \multicolumn{8}{|c|}{ Tempo de duração das disfluências } \\
\hline $0-6$ meses & 0 & 0 & 0 & 0 & 0 & 0 & \multirow{3}{*}{0,346} \\
\hline 6-12 meses & 3 & 9,10 & 2 & 20,00 & 5 & 11,6 & \\
\hline Mais de 12 meses & 30 & 90,90 & 8 & 80,00 & 38 & 88,4 & \\
\hline \multicolumn{8}{|l|}{ Tipologia } \\
\hline Comum & 6 & 18,20 & 2 & 20,00 & 8 & 18,60 & \multirow{3}{*}{0,683} \\
\hline Mista & 6 & 18,20 & 3 & 30,00 & 9 & 21,00 & \\
\hline Gaga & 21 & 63,60 & 5 & 50,00 & 26 & 60,40 & \\
\hline \multicolumn{8}{|c|}{ Fatores estressantes físicos } \\
\hline Ausente & 8 & 24,20 & 9 & 90,00 & 34 & 79,1 & \multirow{2}{*}{0,332} \\
\hline Presente & 25 & 75,80 & 1 & 10,00 & 9 & 20,9 & \\
\hline \multicolumn{8}{|c|}{ Fatores estressantes emocionais } \\
\hline Ausente & 10 & 30,00 & 2 & 20,00 & 12 & 27,90 & \multirow{2}{*}{0,405} \\
\hline Presente & 23 & 70,00 & 8 & 80,00 & 31 & 72,10 & \\
\hline \multicolumn{8}{|c|}{ Fatores comunicativos associados } \\
\hline Ausente & 9 & 27,30 & 3 & 30,00 & 12 & 28,00 & \multirow{3}{*}{$0,003^{*}$} \\
\hline 1 fator & 7 & 21,20 & 6 & 60,00 & 13 & 30,00 & \\
\hline Mais de 1 fator & 17 & 51,50 & 1 & 10,00 & 18 & 42,00 & \\
\hline \multicolumn{8}{|c|}{ Fatores qualitativos associados } \\
\hline Ausente & 4 & 12,10 & 2 & 20,00 & 6 & 14,00 & \multirow{2}{*}{0,794} \\
\hline Presente & 29 & 87,90 & 8 & 80,00 & 37 & 86,00 & \\
\hline
\end{tabular}

Teste de Qui-quadrado, ajustado pela Estatística de Fisher.

*Valores estatisticamente significantes $(p \leq 0,005)$

Tabela 3 - Distribuição das crianças de acordo com a tipologia das disfluências apresentada segundo o tempo de duração das disfluências, fatores qualitativos e comunicativos associados

\begin{tabular}{|c|c|c|c|c|c|c|c|}
\hline \multirow{2}{*}{$\begin{array}{l}\text { Tipologia das } \\
\text { disfluencias }\end{array}$} & \multicolumn{2}{|c|}{$\begin{array}{l}\text { Tempo de duração } \\
\text { das disfluencias }\end{array}$} & \multicolumn{2}{|c|}{$\begin{array}{c}\text { Fatores qualitativos } \\
\text { associados }\end{array}$} & \multicolumn{3}{|c|}{$\begin{array}{c}\text { Fatores comunicativos } \\
\text { associados }\end{array}$} \\
\hline & 6-12m. & $+12 \mathrm{~m}$ & Ausente & Presente & Ausente & 1 fator & $\begin{array}{c}\text { Mais } \\
\text { de } 1 \text { fator }\end{array}$ \\
\hline $\begin{array}{c}\text { Comum } \\
\mathrm{N}=8\end{array}$ & $\begin{array}{c}1 \\
12,50 \%\end{array}$ & $\begin{array}{c}7 \\
87,50 \%\end{array}$ & 0 & $\begin{array}{c}8 \\
100 \%\end{array}$ & 0 & $\begin{array}{c}3 \\
37,50 \%\end{array}$ & $\begin{array}{c}5 \\
62,50 \%\end{array}$ \\
\hline $\begin{array}{l}\text { Mista } \\
\mathrm{N}=9\end{array}$ & $\begin{array}{c}2 \\
22,22 \% \\
\end{array}$ & $\begin{array}{c}7 \\
77,77 \%\end{array}$ & 0 & $\begin{array}{c}9 \\
100 \%\end{array}$ & $\begin{array}{c}2 \\
22,22 \% \\
\end{array}$ & $\begin{array}{c}3 \\
33,44 \%\end{array}$ & $\begin{array}{c}4 \\
44,44 \%\end{array}$ \\
\hline $\begin{array}{l}\text { Gaga } \\
N=26\end{array}$ & $\begin{array}{c}2 \\
7,69 \%\end{array}$ & $\begin{array}{c}24 \\
92,30 \%\end{array}$ & $\begin{array}{c}6 \\
23,07 \%\end{array}$ & $\begin{array}{c}20 \\
76,92 \%\end{array}$ & $\begin{array}{c}10 \\
38,46 \%\end{array}$ & $\begin{array}{c}7 \\
26,92 \%\end{array}$ & $\begin{array}{c}9 \\
34,61 \%\end{array}$ \\
\hline
\end{tabular}


Tabela 4 - Relação entre fatores estressantes físicos e emocionais dos participantes

\begin{tabular}{|c|c|c|c|c|}
\hline \multirow{2}{*}{$\begin{array}{c}\text { Fatores } \\
\text { estressantes } \\
\text { físicos }\end{array}$} & & \multicolumn{3}{|c|}{ Fatores estressantes emocionais } \\
\hline & & Ausente & Presente & Total \\
\hline \multirow{3}{*}{ Ausente } & Freqüência & 10 & 24 & 34 \\
\hline & $\begin{array}{l}\text { Percentual em relação à variável } \\
\text { fatores estressantes físicos ausentes }\end{array}$ & $29,40 \%$ & $70,60 \%$ & $100 \%$ \\
\hline & Percentual em relação ao total & $23,30 \%$ & $55,80 \%$ & $79,10 \%$ \\
\hline \multirow{3}{*}{ Presente } & Freqüência & 2 & 7 & 9 \\
\hline & $\begin{array}{l}\text { Percentual em relação à variável } \\
\text { fatores estressantes físicos presentes }\end{array}$ & $22,20 \%$ & $77,80 \%$ & $100 \%$ \\
\hline & Percentual em relação ao total & $4,7 \%$ & $16,3 \%$ & $20,9 \%$ \\
\hline \multirow{2}{*}{ Total } & Freqüência & 12 & 31 & 43 \\
\hline & Percentual em relação ao total & $27,9 \%$ & $72,1 \%$ & $100 \%$ \\
\hline Valor de $p$ & & 511 & & \\
\hline
\end{tabular}

Teste de Qui-quadrado, ajustado pela estatística de Fisher

${ }^{*}$ Valores estatisticamente significantes $(p \leq 0,005)$

distúrbio, aumenta o risco para a gagueira, como afirmado na literatura "a população com maior risco para a gagueira desenvolvimental poderia ser definida como a criança que está em desenvolvimento de fala, mas especialmente que apresenta um histórico familial de gagueira, que é do gênero masculino e tem algum distúrbio desenvolvimental pré-existente na fala"14.

De acordo com os achados sobre a faixa etária, os resultados apontam que $69,80 \%$ da amostra apresentavam de 5 a 10 anos de idade. Considerando que o início da gagueira freqüentemente ocorre nos anos pré-escolares ${ }^{17-20}$ os dados revelam que os familiares demoraram a procurar o atendimento fonoaudiológico. Esta análise pode ser complementada com as informações sobre o tempo de cronicidade da gagueira, que na maior parte das crianças foi mais de 12 meses de duração $(88,40 \%)$. Portanto, os achados sugerem que crianças disfluentes sem recorrência familial da gagueira, demoraram a procurar o atendimento fonoaudiológico. Neste sentido, ações educativas por parte dos fonoaudiólogos devem ser desenvolvidas para orientar a população sobre a importância de procurar o atendimento fonoaudiológico o mais precocemente possível frente às disfluencias gagas que a criança possa apresentar.

No presente estudo foi verificada uma tendência das meninas apresentarem mais freqüentemente o tipo súbito de início das disfluências em relação aos meninos. Sabe-se que este tipo de início é o menos preocupante para o desenvolvimento da gagueira, conforme estudos prévios ${ }^{11}$. Este dado é coerente com os achados da literatura sobre a maior tendência de ocorrer a recuperação espontânea da gagueira no gênero feminino ${ }^{12,23}$.

A tipologia atípica ou gaga foi a mais predominante na amostra $(60,40 \%)$. Tendo em vista que um dos critérios de inclusão dos participantes era apresentar alto risco para o desenvolvimento da gagueira, este achado era esperado uma vez que estas disfluências são as mais comumente encontradas na gagueira, conforme descrito na literatura ${ }^{24,25}$.

Os dados desta investigação indicam que os fatores qualitativos que acompanham as disfluências, como incoordenação pneumo-fono-articulatória, tensão muscular e taxa de elocução aumentada, ocorreram em grande parte das crianças $(86 \%)$, sugerindo que devem ser valorizados tanto na história clínica como na avaliação fonoaudiológica e podem auxiliar no diagnóstico diferencial da gagueira.

Sendo assim, a análise dos fatores de risco nos casos de gagueira isolada permitiu conhecer que o risco do distúrbio aumenta quando as disfluências duram mais de 12 meses e quando ocorre a presença de fatores qualitativos e comunicativos associados.

A análise dos fatores estressantes que ocorreram nas crianças com alto risco para a gagueira isolada indicou que $79,1 \%$ não manifestaram fatores estressantes físicos. Este achado é discordante de um estudo prévio que acredita que, nos casos de gagueira isolada, fatores que podem ocasionar danos cerebrais precoces ${ }^{9}$ ocorrem mais freqüentemente. 


\section{CONCLUSÃO}

Os resultados desta investigação permitiram concluir que os casos de crianças com alto risco para a gagueira isolada apresentam interação de inúmeros fatores, sugerindo que o distúrbio é multifatorial. Foi possível também concluir que a interação de fatores como gênero masculino, tipologia gaga manifestada por mais de 12 meses, com início persistente, na presença de fatores qualitativos e comunicativos associados pode representar risco maior para o desenvolvimento da gagueira persistente.

A análise do gênero com outros fatores de risco para a gagueira favoreceu a melhor compreensão da natureza complexa do distúrbio, apresentando implicações clínicas principalmente no diagnóstico e prognóstico. Acredita-se que crianças do gênero masculino apresentam um maior risco para a gagueira, e que este risco aumenta na presença de algum outro distúrbio da comunicação associado, independente do histórico familial.

\begin{abstract}
Purpose: to analyze the following factors: gender, age, type of the onset of disfluencies, duration and typology of disfluencies; physical and emotional stresses and communicative and qualitative factors associated in children with high risk for stuttering and without familial recurrence of the disorder. Method: 43 children with high risk for developing mental sporadic stuttering of both genders. Data were gathered through the Protocol of Risk for Stuttering Developmental- PRGD. Results: the ratio male to female was 3.3:1. The occurrence of communicative factors associated in males $(p=0.003)$ was the only statistically significant difference in the analyzed risk factors in male and female. There was a resemblance concerning the findings between male and female: Concerning the duration of the disfluencies, the majority showed more than twelve months of duration, the stuttering-like disfluencies (SLD) were more frequent, the presence of emotional stresses occurred in the majority of the children, and lastly, the qualitative factors associated, such as fast speech rate, tension, and lack of coordination between respiration and phonation were found in most samples. Conclusion: the results of this study suggest that in children with high risk for sporadic stuttering there occurs the interaction of numerous factors, suggesting that this disorder is multifactorial. It was also possible to conclude that the interaction of some risk factors such as male gender, stuttering-like disfluencies (SLD) manifested by more than 12 months with persistent onset, in the presence of communicative and qualitative factors associated may represent increased risk for developing persistent stuttering.
\end{abstract}

KEYWORDS: Speech, Language and Hearing Sciences; Speech; Stuttering; Risk Factors; Speech Disorders

\section{REFERÊNCIAS}

1. Smith A, Kelly E. Stuttering: A dynamic, multifactorial model. In: Curlee RF, Siegel GM. (Org) Nature and treatment of stuttering: New directions. Needham Heights: Allyn \& Bacon. 1997;p. 204-17.

2. Ajdacic-Gross V, Vetter S, Müller M, Kawohl W, Frey $F$, Lupi $G$ et al. Risk factor for stuttering: a secondary analysis of a large data base. Eur Arch Psychiatry Clin Neurosci. 2010; 260(4):279-86.

3. Bothe AK, Davidow JH, Bramlett RE, Ingham RJ. Stuttering treatment research, 1970-2005. I. Systematic review incorporating trial quality assessment of behavioral, cognitive, and related approaches. Am J Speech Lang Pathol. 2006; 15:321-41.
4. Einarsdóttir J, Ingham RJ. The effect of stuttering measurement training on judging stuttering occurrence in preschool children who stutter. J Fluency Disord. 2008; 33:167-79.

5. Dworzynski K, Remington A, Rijsdijk F, Howell P, Plomin R. Genetic etiology in cases of recovered and persistent stuttering in an unselected, longitudinal sample of young twins. Amer J Speech Lang Pathol. 2007; 16:169-78.

6. Brosch S, Winkler S. Diagnostics and therapy of stuttering children. Laryngorhi. 2008; 87(7):519-23.

7. Yairi E. Subtyping stuttering I: A review. J Fluency Disord. 2007; 32:165-96.

8. Oliveira CMC, Souza HA, Santos AC, Cunha D, Giacheti CM. Fatores de risco na gagueira desenvolvimental familial e isolada. 
Rev CEFAC [periódico na Internet]. [acesso em 13 dez 2010]. Disponível em: http://www.scielo. br/scielo.php?script=sci_arttext\&pid=S151618462010005000116\&Ing=en. Prelo 2010. Epub Oct 29, 2010. doi: 10.1590/S1516-18462010005000116. 9. Poulos MG, Webster WG. Family history as a basis for subgrouping people who stutter. J Speech Hear Res. 1991; 34:5-10.

10. Felsenfeld S, Kirk KM, Zhu G, Statham DJ, Neale MC, Martin NG. A study of the genetic and environmental etiology of stuttering in a selected twin sample. Behav Genet. 2000; 5:359-66.

11. Andrade CRF. Gagueira infantil: risco, diagnóstico e programas terapêuticos. Barueri: Pró Fono; 2006.

12. Ambrose NG, Cox NJ, Yairi E. The genetic basis of persistence and recovery in stuttering. J Speech Hear Res. 1997; 40:567-80.

13. Craig A, Hancok K, Tran Y, Craig M, Peters K. Epidemiology of stuttering in the community across the entire life span. J Speech Lang Hear Res. 2002; 45:1097-105.

14. Craig A, Tran Y. The epidemiology of stuttering: The need for reliable estimates of prevalence and anxiety levels over the lifespan. Advan SpeechLang Pathol. 2005; 7:41-6.

15. Yairi E, Ambrose NG. Early childhood stuttering: for clinicians by clinicians. Austin: Pro-Ed; 2005.

16. Yairi E, Ambrose NG. A longitudinal study of stuttering in children: a preliminary report. J Speech Hear Res.1992; 35(4):755-60.

17. Bloodstein O. Some empirical observations about early stuttering: A possibly link to language development. J Commun Disord. 2006; 30:185-91

18. Wagovich SA, Hall NE, Cliffor BA. Speech disruptions in relation to language growth in children who stutter: An exploratory study. J Fluency Disord. 2009; 34(4):242-56.

19. Wittke-Thompson JK, Ambrose N, Yairi EC, Cook EH, Ober C. et al. Genetics studies of stuttering in a founder population J Fluency Disord. 2007; 32:33-50.
20. Bakhtiar M, Seifpanahi S, Ansari H, Ghanadzade $M$, Packman A. Investigation of the reliability of the SSI-3 for preschool Persian-speaking children who stutter. J Fluency Disord. 2010; 35(2):87-91.

21. Reilly $S$, Onslow $M$, Packman $A$, Wake $M$, Bavin EL, Prior M, Eadie P, Cini E, Bolzonello C, Ukoumunne OC. Predicting stuttering onset by age of 3 years: A prospective, community cohort study. Pediatrics. 2009; 123:270-7.

22. Logan KJ, Mullins MS, Jones KM. The depiction of stuttering in contemporary juvenile fiction: implications for clinical practice. Psychol Schools. 2008; 45(7):609-26.

23. Yairi E, Ambrose NG, Paden EP, Throneburg RN. Predictive factors of persistence and recovery: pathways of childhood stuttering. J Fluency Disord. 1996; 29:51-77.

24. Sawyer J, Yairi E. The effect of sample size on the assessment of stuttering severity. Am J Speech Lang Pathol. 2006; 15:36-44.

25. Suresh R, Ambrose N, Roe C, Pluzhnikov A, Wittke-Thompson JK, Ng MCY. et al.New complexities in the genetivs of stuttering: Significant sex-specific linkage signals. Am J Hum Genet. 2006; 78:554-63.

26. Smith A, Sadagopan N, Walsh B, Weber-Fox C. Increasing phonological complexity reveals heightened instability in inter-articulatory coordination in adults who stutter. J Fluency Disord. 2010; 35:1-18.

27. Craig A, Tran Y. Chronic and social anxiety in people who stutter. Adv Psychiat Treat. 2006; 12:63-8.

28. Andrews G, Graig A, Feyer AM, Hoddinott S, et al. (1983). Stuttering: a review of research findings and theories circa 1982. J Speech Hear Disord. 1983; 48:226-46.

29. Bloodstein O. A handbook on stuttering. Chicago: National Easter Seal Society, 1995.
http://dx.doi.org/10.1590/S1516-18462011005000062

RECEBIDO EM: 29/12/2010

ACEITO EM: 23/03/2011

Endereço para correspondência:

Cristiane Moço Canhetti de Oliveira

Av. Hygino Muzzi Filho, 737, Vila Universitária

Marília - SP

CEP: $17525-000$

E-mail: cmcoliveira@terra.com.br 